\title{
Measurements and combinations of effective weak angle in Drell-Yan di-electron and di-muon channels at CDF and D0
}

Willis Sakumoto*†

University of Rochester

E-mail: willis@fnal.gov

CDF and D0 have measured the effective leptonic weak mixing angle $\sin ^{2} \theta_{\text {eff }}^{\text {lept }}$, using their full Tevatron datasets. The preliminary combination of these results yields $\sin ^{2} \theta_{\text {eff }}^{\text {lept }}=0.23179 \pm 0.00035$, where the uncertainty includes both statistical and systematic uncertainties [1]. Within the standard model (SM) context of ZFITTER weak radiative corrections, the inference of the electroweak mixing angle $\sin ^{2} \theta_{W}$ (or equivalently, the $W$-boson mass) gives $0.22356 \pm 0.00035$ (or equivalently $80.351 \pm 0.018 \mathrm{GeV} / c^{2}$ ).

38th International Conference on High Energy Physics 3-10 August 2016

Chicago, USA

\footnotetext{
* Speaker.

${ }^{\dagger}$ Presented on behalf of the CDF and D0 collaborations
} 
At the Fermilab Tevatron proton-antiproton $(p \bar{p})$ collider, Drell-Yan lepton pairs are produced in the process $p \bar{p} \rightarrow \ell^{+} \ell^{-}+X$. At the Born level, there are two parton-level processes $q \bar{q} \rightarrow \gamma^{*} \rightarrow \ell^{+} \ell^{-}$and $q \bar{q} \rightarrow Z \rightarrow \ell^{+} \ell^{-}$, where the $q$ and $\bar{q}$ are the quark and antiquark, respectively, from the colliding hadrons. The photon-fermion interaction is via a vector current, and the coupling strength is the fermion $(f)$ charge $Q_{f}$. $Z$ boson-fermion interactions are via both vector and axial-vector currents, and the coupling strengths are $g_{V}^{f}=T_{3}^{f}-2 Q_{f} \sin ^{2} \theta_{W}$ and $g_{A}^{f}=T_{3}^{f}$, respectively, where $T_{3}^{f}$ is the third component of the fermion weak-isospin. The interference of the axial-vector current amplitude with the vector current amplitudes (of the photon and $Z$ boson) induces an asymmetry in the polar angle $\vartheta$ distribution of lepton pairs in their rest frame: $1+\cos ^{2} \vartheta+A_{4} \cos \vartheta . A_{4}$ is directly related to $\sin ^{2} \theta_{W}$. Weak radiative corrections alter the Born-level $Z$ couplings. ZFITTER [2] SM corrections, which are organized into complex valued form-factors, turn $\sin ^{2} \theta_{W}$ into slightly differing effective-mixing angles for leptons, $u$-type, and $d$-type quarks. $A_{4}$ is most sensitive to the leptonic effective-mixing angle, denoted by $\sin ^{2} \theta_{\text {eff }}^{\text {lept }}$. In all orders of the on-shell renormalization scheme [3] used by ZFITTER, $\sin ^{2} \theta_{W}=1-M_{W}^{2} / M_{Z}^{2}$, where $M_{W}$ and $M_{Z}$ are the $W$ and $Z$ boson masses, respectively. As the $Z$-boson mass is accurately known, the inference of $\sin ^{2} \theta_{W}$ from effective-mixing angle measurements is also an indirect $W$-boson mass measurement.

The decay angles are measured in the Collins-Soper (CS) rest frame [4] shown in Fig. 1left. It is reached from the laboratory frame via a longitudinal boost along the beam line to
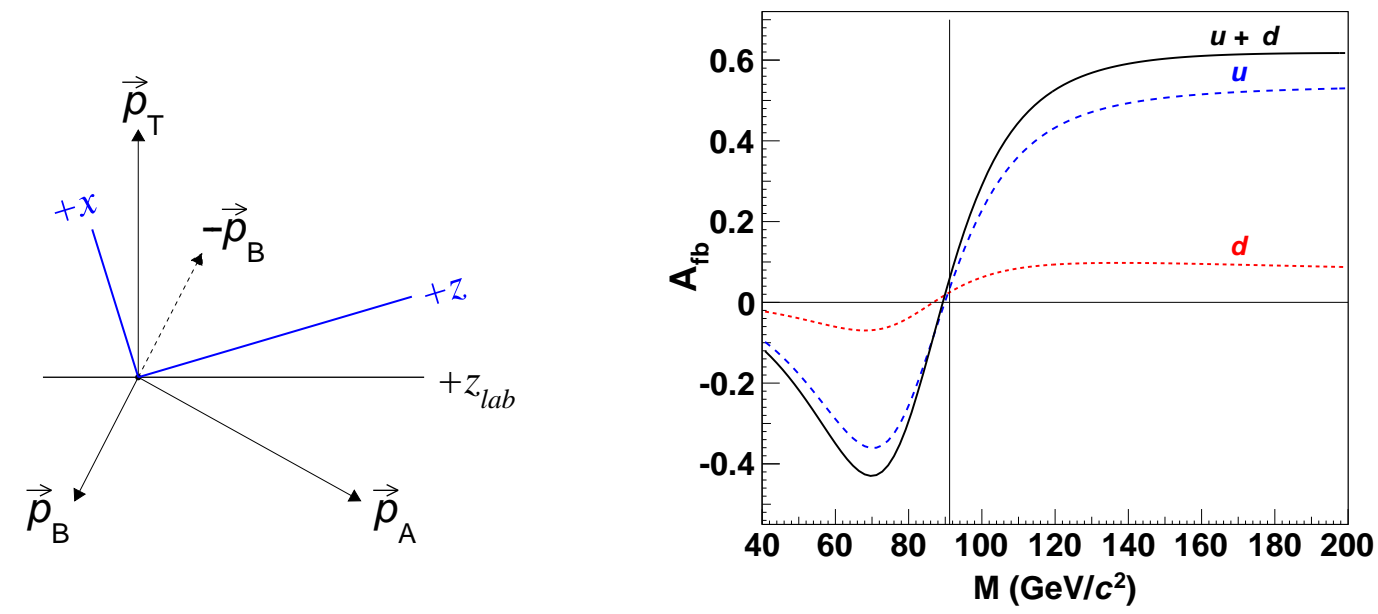

Figure 1: Left) Collins-Soper frame. In the laboratory frame, the proton is along $z_{\text {lab }}$, and $\vec{P}_{\mathrm{T}}$ is the lepton-pair transverse momentum. The proton and antiproton momentum vectors in the rest frame are $\vec{P}_{\mathrm{A}}$ and $\vec{P}_{\mathrm{B}}$, respectively. The CS $z$ axis is the angular bisector of $\vec{P}_{\mathrm{A}}$ and $-\vec{P}_{\mathrm{B}}$. The CS $y$ axis is in the direction of $\vec{P}_{\mathrm{B}} \times \vec{P}_{\mathrm{A}}$. The CS $x$ axis is in the direction away from $\vec{P}_{\mathrm{A}}+\vec{P}_{\mathrm{B}}$. Right) $A_{\mathrm{fb}}(M): u+d$ denotes the overall asymmetry, with $u$ and $d$ denoting the contributions to the overall asymmetry from quarks with charge $2 / 3$ and $-1 / 3$, respectively, so $A_{\mathrm{fb}}(u+d)=A_{\mathrm{fb}}(u)+A_{\mathrm{fb}}(d)$. The vertical line is $M=M_{Z}$.

frame where the lepton-pair longitudinal momentum is zero, followed by a transverse boost along the lepton-pair transverse momentum to the rest frame. The $\ell^{-}$forward-backward 
asymmetry in $\cos \vartheta$ is defined as $A_{\mathrm{fb}}(M)=\left[\sigma^{+}(M)-\sigma^{-}(M)\right] /\left[\sigma^{+}(M)+\sigma^{-}(M)\right]$, where $M$ is the lepton-pair mass, $\sigma^{+}$the total cross section for $\cos \vartheta \geq 0$, and $\sigma^{-}$the total cross section for $\cos \vartheta<0$. $A_{\mathrm{fb}}(M)$ equals $\frac{3}{8} A_{4}(M)$. Figure 1-right shows the typical dependence of the asymmetry as a function of the lepton-pair mass. The offset of $A_{\mathrm{fb}}$ from zero at $M=M_{Z}$ is directly related to $\sin ^{2} \theta_{\text {eff }}^{\text {lept }}$. Away from the $Z$ pole, $A_{\mathrm{fb}}(M)$ is dominated by the component from $\gamma^{*}-Z$ interference, which is not directly related to the mixing angle but sensitive to the flux of partons. $A_{\mathrm{fb}}(M)$ constrains both the mixing angle and the parton distribution functions (PDF) of the proton.

The D0 asymmetry measurement is based on $e^{+} e^{-}$pairs [5]; the muon channel measurement was not available. Electrons of $P_{\mathrm{T}}>25 \mathrm{GeV} / c$ from the central calorimeter (CC) with $|\eta|<1.1$ and forward calorimeter (EC) with $1.5<|\eta|<3.2$ are used. The asymmetry is measured separately in all electron-pair topologies CC-CC, CC-EC, and ECEC, where there are 248000,241000 , and 71000 events, respectively. Backgrounds are removed from the data. The $W+$ jets, $\gamma^{*} / Z \rightarrow \tau \tau, W W$ and $W Z$ and $t \bar{t}$ backgrounds are estimated with PYTHIA 6.2 [6]. QCD backgrounds are estimated using the data. The mixing angle is separately extracted for each topology using templates with varying values of a single parameter $\sin ^{2} \theta_{\text {eff }}^{\text {lept }}$, and then combined. These templates, calculated with the PYTHIA 6.2 and the NNPDF 2.3 [7] PDFs, are reweighted to incorporate QCD higher-order effects, and include detector simulation. As the result is baised because the leptonic and quark effective-mixing angles differ in value, a bias correction of +0.00008 estimated with ZGRAD [8] and RESBOS [9] is applied to the result, yielding $\sin ^{2} \theta_{\text {eff }}^{\text {lept }}=0.23147 \pm 0.00043$ (stat.) \pm 0.00017 (PDF) \pm 0.00008 (syst.). The systematic uncertainty is for energy calibration, energy smearing, backgrounds, charge misidentification, and electron misidentification. The PDF uncertainty is obtained using the 100 equally probable ensemble PDFs of NNPDF 2.3.

The CDF asymmetry measurement uses muons with $P_{\mathrm{T}}>20 \mathrm{GeV} / c$ and $|\eta|<1.1$, and electrons from the central $(\mathrm{C})$ calorimeter region with $0.05<|\eta|<1.05$, and endplug (P) calorimeter region with $1.2<|\eta|<2.8$ [10]. As the end-plug region tracking volume is significantly reduced, electron pairs contain a candidate from the central region, where tracks are well measured. There are $227000 \mathrm{CC}$-topology $e^{+} e^{-}$pairs with $E_{\mathrm{T}}>25 / 15 \mathrm{GeV}$, and $250000 \mathrm{CP}$-pairs with $E_{\mathrm{T}}>20 \mathrm{GeV}$. There are $277000 \mu^{+} \mu^{-}$ pairs. Backgrounds are removed from the data. The $W+$ jets, $\gamma^{*} / Z \rightarrow \tau \tau, W W, W Z$ and $Z Z$, and $t \bar{t}$ backgrounds are estimated with PYTHIA 6.2. QCD backgrounds are estimated using the data. The measurements are corrected for direct fits to calculated templates. The data-driven event-weighting method [11] provides the first-order correction to the acceptance and efficiency. Both measurements and calculations are restricted to rapidity regions with sufficient acceptance: $|y|<1$ for muon pairs, and $|y|<1.7$ for electron pairs. The simulation, used to unfold detector and QED FSR smearing effects, and to remove residual biases of a few percent, consists of PYTHIA 6.2 [6] event generation, simulation of QED final-state radiation (FSR) via PHOTOS 2.0 [12], and detector simulation. Higher order QCD effect corrections are applied. Templates for the electron and muon channels are calculated using the same POWHEG-BOX NLO Drell-Yan framework [13] with NNPDF-3.0 [14] NNLO PDFs, followed by PYTHIA 6.41 [15] parton shower- 
ing. ZFITTER complex-valued form-factor functions are incorporated for fermion-type dependent mixing angles. The template fits yield $\sin ^{2} \theta_{\text {eff }}^{\text {lept }}=0.23141 \pm 0.00086$ (stat.) and $0.23248 \pm 0.00049$ (stat.) for the muon and electron channels, respectively. The combined result is $0.23221 \pm 0.00043$ (stat.) \pm 0.00006 (syst.) \pm 0.00016 (PDF). The systematic uncertainty is from the energy scale and resolution, backgrounds, and QCD scale. The PDF uncertainty is obtained using the 100 equally probable ensemble PDFs of NNPDF 3.0.

Prior to the CDF and D0 result combination, the D0 result, which uses NNPDF-2.3 PDFs and ZGRAD plus RESBOS based weak corrections, is standardized to a common baseline with CDF using NPDF-3.0 PDFs and ZFITTER SM weak corrections. Standardization offsets to the D0 result for $\sin ^{2} \theta_{\text {eff }}^{\text {lept }}$ are evaluated and applied. The PDF correction is determined with $A_{\mathrm{fb}}$ pseudodata based on the default NNPDF-3.0 PDF and a reference value for $\sin ^{2} \theta_{\text {eff }}^{\text {lept }}$, and templates from NNPDF-2.3 with varying values of $\sin ^{2} \theta_{\text {eff }}^{\text {lept }}$. The PDF offset, the difference between the reference and the template-fit value, is $-0.00024 \pm$ 0.00004 , where the uncertainty is statistical. The weak-correction offset is the difference between $\sin ^{2} \theta_{\text {eff }}^{\text {lept }}$ derived from the CDF $A_{\mathrm{fb}}$ measurement and templates with and without ZFITTER corrections. Its value is $+0.00014 \pm 0.00004$, where the uncertainty is statistical. The preliminary corrected D0 result is $\sin ^{2} \theta_{\text {eff }}^{\text {lept }}=0.23137 \pm 0.00043$ (stat.) \pm 0.00019 (syst.), where the systematic uncertainty includes the standardization uncertainty of \pm 0.00005 ; while the input D0 result is published, the correction is preliminary.

The CDF muon- and electron-channel combined result and the standardized D0 results for $\sin ^{2} \theta_{\text {eff }}^{\text {lept }}$ are combined using the "Best Linear Unbiased Estimate" method [16]. The PDF uncertainties are treated as $100 \%$ correlated. The statistical uncertainties, other systematic uncertainties, and the standardization uncertainty of the D0 result are treated as uncorrelated. The combination result is $\sin ^{2} \theta_{\text {eff }}^{\text {lept }}=0.23179 \pm 0.00030$ (stat.) \pm 0.00006 (syst.) \pm $0.00017(\mathrm{PDF})$, where the second uncertainty is the quadrature combination of the uncorrelated and correction systematic uncertainties. For this combination, the resulting statistical, uncorrelated systematic, D0 standardization, and PDF uncertainties are \pm 0.00030 , $\pm 0.00005, \pm 0.00003$, and \pm 0.00017 , respectively. The combination weights are approximately equal, and the $\chi^{2}$ is 1.8 .

In the context of the $\mathrm{SM}, \sin ^{2} \theta_{W}$ and the $W$-boson mass are inferred from $\sin ^{2} \theta_{\text {eff }}^{\text {lept }}$ with the aid of ZFITTER: $\sin ^{2} \theta_{\text {eff }}^{\text {lept }}=\operatorname{Re}\left[\kappa_{\ell}\left(M_{Z}^{2}, \sin ^{2} \theta_{W}\right)\right] \sin ^{2} \theta_{W}$, where $\kappa_{\ell}$ is the leptonic form factor, which depends on SM input parameters. The value of $\operatorname{Re}\left[\kappa_{\ell}\right]$ at the $Z$-pole mass is approximately 1.037, and is most sensitive to the top-quark mass. With a mass value of $173.2 \pm 0.9 \mathrm{GeV} / c^{2}$ [17], the uncertainty is \pm 0.00008 . A Higgs mass value of $125 \mathrm{GeV} / c^{2}$ is used in the calculation. The inferences for the CDF measurement are $\sin ^{2} \theta_{W}=0.22400 \pm$ $0.00041 \pm 0.00019$ and $M_{W}$ (indirect) $=80.328 \pm 0.021 \pm 0.010 \mathrm{GeV} / c^{2}$. For the corrected measurement of D0, the preliminary results are $\sin ^{2} \theta_{W}=0.22313 \pm 0.00041 \pm 0.00020$ and $M_{W}$ (indirect) $=80.373 \pm 0.021 \pm 0.010 \mathrm{GeV} / c^{2}$. The preliminary inference results for the $\mathrm{CDF}$ and D0 combination are $\sin ^{2} \theta_{W}=0.22356 \pm 0.00029 \pm 0.00019$ and $M_{W}$ (indirect) $=$ $80.351 \pm 0.015 \pm 0.010 \mathrm{GeV} / c^{2}$. For all inferences, the first contribution to the uncertainties is statistical and the second is systematic. All systematic uncertainties are combined in quadrature, including the form-factor uncertainty.

The measurements of $\sin ^{2} \theta_{\text {eff }}^{\text {lept }}$ are compared with previous results from the Tevatron, 
LHC, LEP-1, and SLC in Fig. 2-left. The hadron collider results are from $A_{\mathrm{fb}}$ measure-
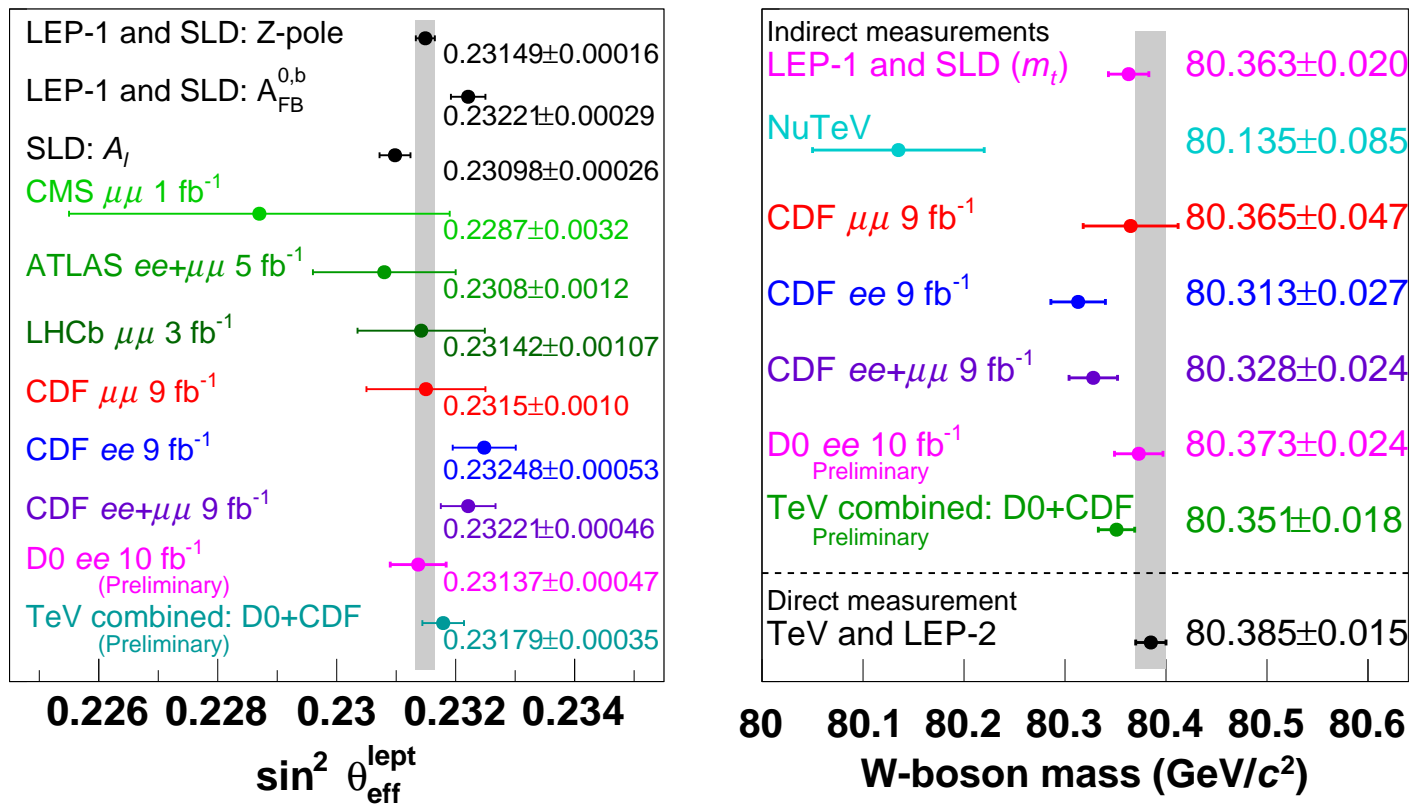

Figure 2: The CDF and D0 combination results are denoted as "TeV combined: D0 + CDF", and the updated D0 electron channel results by "D0 ee $10 \mathrm{fb}^{-1}$ ". The horizontal bars represent total

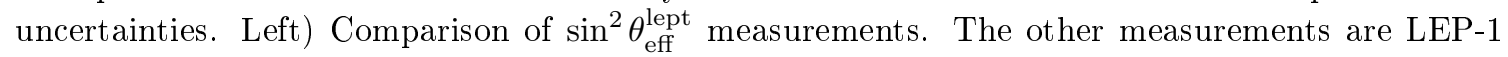
and SLD [18], CMS [19], ATLAS [20], LHCb [21], and CDF [10]. Right) Comparison $W$-boson mass measurements. The other indirect measurements are from LEP-1 and SLD [18, 22] which includes the Tevatron top-quark mass measurement [17], NuTeV [23], and CDF [10]. The direct measurement is from the Tevatron and LEP-2 [24].

ments. The LEP-1 and SLD results are from asymmetry measurements at the $Z$ pole [18]. The $W$-boson mass inferences are compared in Fig. 2-right with previous direct and indirect measurements from the Tevatron, NuTeV, LEP-1, SLD, and LEP-2. The indirect measurement from LEP-1 and SLD, based on $\sin ^{2} \theta_{W}=0.22332 \pm 0.00039$, is from the SM fit to all $Z$-pole measurements described in Appendix $\mathrm{F}$ of Ref. [22]. The following input parameters to ZFITTER, the Higgs-boson mass, the $Z$-boson mass, the QCD coupling at the $Z$ pole, and the light-quark correction to $\alpha_{\mathrm{em}}$ at the $Z$ pole are varied simultaneously within the constraints of the LEP-1 and SLD data, while the top-quark mass is constrained to the directly measured value from the Tevatron, $173.2 \pm 0.9 \mathrm{GeV} / c^{2}$ [17].

The new Tevatron measurement on the effective-leptonic weak-mixing parameter is the most precise from hadron colliders. With the inclusion of ZFITTER weak radiative corrections, the underlying $\sin ^{2} \theta_{W}$ and the $W$-boson mass are inferred within the context of the standard model as well.

\section{References}

[1] T. Aaltonen et al. (CDF and D0 Collaboration), FERMILAB-CONF-16-256-E (Aug. 2016), http://tevewwg.fnal.gov/wz/sw2eff/. 
[2] D. Bardin, M. Bilenky, T. Riemann, M. Sachwitz, and H. Vogt, Comput. Phys. Commun. 59, 303 (1990); D. Bardin, P. Christova, M. Jack, L. Kalinovskaya, A. Olchevski, S. Riemann, and T. Riemann, Comput. Phys. Commun. 133, 229 (2001); A. Arbuzov, M. Awramik, M. Czakon, A. Freitas, M. Grünewald, K. Monig, S. Riemann, and T. Riemann, Comput. Phys. Commun. 174, 728 (2006).

[3] A. Sirlin, Phys. Rev. D 22, 971 (1980).

[4] J.C. Collins and D.E. Soper, Phys. Rev. D16, 2219 (1977).

[5] V. M. Abazov et al. (D0 Collaboration), Phys. Rev. Lett. 115, 041801 (2015); V. M. Abazov et al. (D0 Collaboration), Phys. Rev. D 84, 012007 (2011).

[6] T. Sjöstrand, P. Edén, L. Lönnblad, G. Miu, S. Mrenna, and E. Norrbin, Comput. Phys. Commun. 135, 238 (2001).

[7] R.D. Ball et al. (NNPDF Collaboration), Nucl. Phys. B867, 244 (2013).

[8] U. Baur, O. Brein, W. Hollik, C. Schappacher, and D. Wackeroth, Phys. Rev. D 65, 033007 (2002).

[9] G.A. Ladinsky and C.-P. Yuan, Phys. Rev. D50 4239 (1994); C. Balazs and C.-P. Yuan, Phys. Rev. D56, 5558 (1997); F. Landry, R. Brock, P.M. Nadolsky, and C.-P. Yuan, Phys. Rev. D67 073016 (2003).

[10] T. Aaltonen et al. (CDF Collaboration), Phys. Rev. D 89, 072005 (2014); T. Aaltonen et al. (CDF Collaboration), Phys. Rev. D 93, 112016 (2016).

[11] A. Bodek, Eur. Phys. J. C 67, 321 (2010).

[12] E. Barberio and Z. Was, Computer Phys. Comm. 79, 291 (1994); E. Barberio, B. van Eijk, and Z. Was, Computer Phys. Comm. 66, 115 (1991); P. Golonka and Z. Was, Eur. Phys. J. C 45, 97 (2006).

[13] S. Alioli and P. Nason and C. Oleari and E. Re, J. High Energy Phys. 07, 060 (2008); S. Frixione and P. Nason and C. Oleari, J. High Energy Phys. 11, 070 (2007).

[14] R.D. Ball, V. Bertone et al. (NNPDF Collaboration), J. High Energy Phys. 04 (2015) 40.

[15] T. Sjöstrand, S. Mrenna, and P. Z. Skands, J. High Energy Phy. 05, (2006) 026.

[16] L. Lyons, D. Gibault, and P. Cliffort, Nucl. Instrum. Methods Phys. Res. Sect. A 270, 110 (1988).

[17] T. Aaltonen et al. (CDF and D0 Collaborations), Phys. Rev. D 86, 092003 (2012).

[18] The ALEPH, DELPHI, L3, OPAL, SLD Collaborations, the LEP Electroweak Working Group, the SLD Electroweak and Heavy Flavour Groups, Phys. Rept. 427, 257 (2006).

[19] S. Chatrchyan et al. (CMS Collaboration), Phys. Rev. D 84, 112002 (2011).

[20] G. Aad et al. (ATLAS Collaboration), J. High Energy Phys. 09, 049 (2015).

[21] R. Aaij et al. (LHCb Collaboration), J. High Energy Phys. 11, 190 (2015).

[22] The ALEPH, DELPHI, L3, OPAL Collaborations, the LEP Electroweak Working Group, Phys. Rept. 532, 119 (2013).

[23] G. P. Zeller et al. (NuTeV Collaboration), Phys. Rev. Lett. 88, 091802 (2002); Phys. Rev. Lett. 90, 239902(E) (2003).

[24] T. Aaltonen et al. (CDF and D0 Collaboration), Phys. Rev. D 88, 052018 (2013). 